\title{
INEQUALITIES RELATING DIFFERENT DEFINITIONS OF DISCREPANCY
}

Dedicated to the memory of Hanna Neumann

C. J. SMYTH

(Received 27 June 1972)

Communicated by M. F. Newman

\section{Introduction}

Let $\boldsymbol{p}_{1}, \boldsymbol{p}_{2}, \cdots, \boldsymbol{p}_{N}$ be $N$ points in the unit $s$-dimensional closed square $Q=[0,1]^{s}$. For any measurable set $S \subseteq Q$, we define $\delta(S)$, the discrepancy of $S$, by

$$
\delta(S)=V(S)-n(S) / N,
$$

where $V(S)$ is the $s$-dimensional volume of $S$, and $n(S)$ is the number of indices $i$ for which $p_{i} \in S$. Let

$$
D_{K}=\sup |\delta(B)|,
$$

where the supremum is taken over all $s$-balls $B \subseteq Q$, and

$$
D_{c}=\sup |\delta(C)| \text {, }
$$

the supremum in this case being taken over all convex sets $C \subseteq Q$. Clearly $D_{c} \geqq D_{K}$. In this paper we establish

THEOREM 1. $D_{K} \geqq \phi_{1}(s) D_{c}^{s+1}$, where $\phi_{1}$ is a constant depending only on $s$.

This replaces the best previously known inequality in this direction,

$$
D_{K} \geqq \exp \left(-\gamma_{1} D_{c}^{-\gamma_{2}}\right)
$$

$\left(\gamma_{1}, \gamma_{2}\right.$ depending only on $\left.s\right)$ due to Hlawka [3].

In the other direction, I have given an example in [3] to show that the exponent in Theorem 1 cannot be improved to less than $\frac{1}{2}(s+1)$.

Denote by $D$ the classical $s$-interval discrepancy $D=\sup |\delta(J)|$, the supremum being taken over all intervals $J$ of the form

$$
J=\left\{\boldsymbol{x}=\left(x_{j}\right) \mid 0 \leqq \alpha_{j} \leqq x_{j}<\beta_{i} \leqq 1(j=1, \cdots, s)\right\} .
$$


Cassels (unpublished) has proved

$$
D_{K} \geqq \phi_{2}(s) D^{s+1} ;
$$

my proof of Theorem 1 is a fairly natural generalisation, from intervals to convex bodies, of his proof of (2). Recently I [3] have improved (2) to $D_{K} \geqq \phi_{3}(s, \varepsilon) D^{s+\varepsilon}$. Hlawka [2] has proved the inequality (5) below relating $D$ and $D_{c}$ (see also $[3]$ ). Thus we have the following six inequalities connecting $D_{K}, D_{c}$ and $D$ :

$$
\begin{aligned}
D_{c} & \geqq D \\
D_{c} & \geqq D_{K} \\
D & \geqq \phi_{4}(s) D_{c}^{s} \\
D & \geqq \phi_{4}(s) D_{K}^{s} \\
D_{K} & \geqq \phi_{1}(s) D_{c}^{s+1} \\
\text { for any } \varepsilon>0, D_{k} & \geqq \phi_{3}(s, \varepsilon) D^{s+\varepsilon} .
\end{aligned}
$$

$\left(\phi_{1}, \phi_{4}\right.$ depend only on $s, \phi_{3}$ only on $s$ and $\varepsilon$ ).

Of these inequalities (3) and (4) are, of course, trivial while (6) follows straight from (4) and (5),

We give a proof of Theorem 1 in section 3 . Section 2 contains preliminaries needed for the proof,

I would like to thank Professor Cassels for suggesting this problem to me, and for making available his unpublished proof of (2).

\section{Preliminaries}

Before proving the theorem, we need some more notation, and two lemmas relating to convex bodies.

First of all, since Theorem 1 is trivial when $s=1$, we always assume that $s \geqq 2$. We let $\theta_{s}=\pi^{s / 2} / \Gamma(s / 2+1)$ be the volume of the unit $s$-ball, and $B_{r}(y)$ be the closed ball with centre $y$, radius $r$. Denote by $\chi_{y}$ the characteristic function of $B_{r}(y)\left(=1\right.$ if $x \in B_{r}(y),=0$ if not).

Let $C$ be any convex set. For any $r>0$ we denote by $C^{r}$ the set

$$
C^{r}=\{x+y|x \in C,| y \mid \leqq r\}
$$

and by $C_{r}$ the set of points of $C$, distant greater than $r$ from every point outside $C$. Clearly $C_{r} \subseteq C \subseteq C^{r}$. We have an expression for the volume of $C^{r}$, given by

Lemma 1. For any convex set $C$,

$$
V\left(C^{r}\right)=V(C)+A_{1}(C) r+A_{2}(C) r^{2}+\cdots+A_{s}(C) r^{s},
$$

where $A_{1}, A_{2}, \cdots, A_{s}$ are constants ("mixed volumes") depending only on $C$, with 
the property that if $C \subseteq C^{\prime} C^{\prime}$ convex, then

$$
A_{\imath}(C) \leqq A_{i}\left(C^{\prime}\right) . \quad(i=12, \cdots, s)
$$

The coefficient $A_{1}(C)$ is the $(s-1)$-dimens:onal area of the boundary of $C$. Furthermore, if also $C \subseteq Q$, then

$$
V\left(C^{r}\right)-V(C) \leqq 2 s r(1+2 r)^{s-1} .
$$

Proof. Equations (10) and (11) are special cases of Theorems 41 and 42 respectively in Eggleston's Cambridge Tract [1], and the expression for $A_{1}(C)$ as as the surface are of $C$ is on p. 88 of [1]. It therefore remains only to deduce the inequality (12). Now $C \subseteq Q$, so

$$
A_{i}(C) \leqq A_{i}(Q) \quad(i=1,2, \cdots, s)
$$

by (11). Hence from (10),

But

$$
V\left(C^{r}\right)-V(C) \leqq V\left(Q^{r}\right)-V(Q)=V\left(Q^{r} \backslash Q\right)
$$

$$
V(Q \backslash Q) \leqq(1+2 r)^{s}-1 \leqq 2 r s(1+2 r)^{s-1}
$$

by the mean value theorem. This completes the proof of Lemma 1 .

Also, for $C_{r}$, we have

Lemma 2. For any convex set $C \subseteq Q$,

$$
V(C)-V(C) \leqq r A_{1}(C) \leqq 2 r s .
$$

Proof. For a convex polytope $P$, the first inequality becomes clear by mounting a cylinder of height $r$, facing inwards, on each face of $P$. We now consider the case of a general convex body $C$. By [1] (Theorem 33), for each $\varepsilon>0$ there is a convex polytope $P$ such that

$$
P \subseteq C \subseteq P^{\varepsilon} \text {. }
$$

So

$$
\begin{aligned}
V\left(C \backslash C_{r}\right) & \leqq V\left(P \backslash P_{r}\right)+V\left(P^{8} \backslash P\right) \\
& \leqq r A_{1}(P)+2 s \varepsilon(1+2 \varepsilon)^{s-1}
\end{aligned}
$$

by (13) for $p$ and (12)

$$
\leqq r A_{1}(C)+2 s \varepsilon(1+2 \varepsilon)^{s-1}
$$

by (11). Hence on letting $\varepsilon \rightarrow 0$, we obtain the first part of (13).

The second part of (13) follows from the fact that $A_{1}(C) \leqq A_{1}(Q)=2$ s. This proves the lemma.

\section{Proof of Theorem 1}

We are now ready to prove Theorem 1 . We take a convex body $C \subseteq Q$, which we can assume has $\delta(C) \neq 0$. We must produce a ball $B$ with 


$$
|\delta(B)| \geqq \phi_{1}|\delta(C)|^{s+1} \text {. }
$$

The method of the proof is to use Cassels' idea of averaging the discrepancies of circles of fixed radius, as the centre varies throughout the given body $C$.

We first show (equation (14)) that, apart from the boundary effects, the integral of $\delta\left(B_{r}(y)\right)$ over $y \in C$ is roughly $\theta_{s} s \delta(C)$. Ignoring boundary effects, we then have

$$
\left|\delta\left(B_{r}(y)\right)\right| V(C) \geqq \theta_{s} r^{s}|\delta(C)|
$$

for some $y \in C$. It is then only a question of choosing $r$ as large as one can, without the boundary effects becoming too significant, and we obtain Theorem 1. I now give the details.

We first show that for any $r>0$,

$$
\int_{C} \delta\left(B_{r}(y) \cap C_{r}\right) d y=\theta_{s} r^{s} \delta\left(C_{r}\right)
$$

Now as $\delta$ is a signed measure,

$$
\begin{aligned}
\int_{C} \delta\left(B(y) \cap C_{r}\right) d y & =\int_{C} \int_{\left(B_{r}(y) \cap C_{r}\right)} d \delta(x) d y \\
& =\int_{C} \int_{C_{r}} \chi_{y}(x) d \delta(x) d y \\
& =\int_{C} \int_{C_{r}} \chi_{x}(y) d \delta(x) d y \\
& =\int_{C^{r}} \int_{C} \chi_{x}(y) d y d \delta(x) \\
& =\int_{C_{r}} V\left(B_{r}(x) \cap C\right) d \delta(x) \\
& =\theta_{s} r^{s} \int_{C_{r}} d \delta(x)=\theta_{s} r^{s} \delta\left(C_{r}\right)
\end{aligned}
$$

as $B_{r}(x) \subseteq C$ for $x \in C_{r}$. This proves (14).

We now separate the three cases I: $\delta(C)>0$, II: $\delta(C)<0, C^{2 r} \subseteq Q$, III: $\delta(C)<0, C^{2 r} \notin Q$, where in II and III $r=-\delta(C) / 16$ s.

CASE I. $\delta(C)>0$. For $y \in C_{2 r}$, we have $B_{r}(y) \subseteq C$, and hence

$$
B_{r}(y)=B_{r}(y) \cap C .
$$

Using (14), we obtain

$$
\int_{C_{2 r}} \delta\left(B_{r}(y)\right) d y=\theta_{s} r \delta\left(C_{r}\right)-\int_{\left(C \backslash C_{2 r}\right)} \delta\left(B_{r}(y) \cap C_{r}\right) d y
$$




$$
\begin{aligned}
& =\theta_{s} r^{s} \delta(C)-\theta_{s} r^{s} \delta\left(C \backslash C_{r}\right)-\int_{\left(C \backslash C_{2 r}\right)} \delta\left(B_{r}(y) \cap C_{r}\right) d y \\
& \geqq \theta_{s} r^{s} \delta(C)-\theta_{s} r^{s} V\left(C \backslash C_{r}\right)-\int_{\left(C_{C} C_{2} r\right)} V\left(B_{r}(y) \cap C_{r}\right) d y
\end{aligned}
$$

as $\delta(S) \leqq V(S)$ for any set $S$,

$$
\begin{aligned}
& \geqq \theta_{s} r\left\{\delta(C)-V\left(C \backslash C_{r}\right)-V\left(C \backslash C_{2 \mathrm{r}}\right)\right\} \\
& \geqq \theta_{s} r^{s}(\delta(C)-6 r s)
\end{aligned}
$$

by Lemma 2. So we choose $r=\delta(C) / 12 s$. Then

$$
\int_{C_{2 r}} \delta\left(B_{r}(y)\right) d y \geqq \frac{\theta_{s} s^{s}}{2} \cdot \delta(C)=\frac{\theta_{s}}{2} \frac{(\delta(C))^{s+1}}{(12 s)^{s}} .
$$

Hence there is some $y \in C_{2 r}$ such that

$$
V\left(C_{2 r}\right) \delta\left(B_{r}(y)\right) \geqq \frac{\theta_{s}}{2} \frac{(\delta(C))^{s+1}}{(12 s)^{s}} .
$$

Since $V\left(C_{2 r}\right) \leqq V(C) \leqq 1$, we now have Theorem 1 as required.

CASE II. $\delta(C)<0, C^{2 r} \subseteq Q$, where $r=(-\delta(C)) / 16$ s. We write $\delta(S)=-\delta(S)$.

Firstly, it is clear that the convex subset of $C$ with $\delta$ maximal is the convex hull of some subset of the points $p_{1}, p_{2}, \cdots, p_{N}$. Hence, replacing $C$ by this set if necessary, we can assume that, both for cases II and III,

$$
\bar{\delta}\left(C^{\prime}\right) \leqq \delta(C)
$$

for all convex subsets $C^{\prime}$ of $C$.

Now it is easy to verify that $\left(C^{r}\right)_{r}=C$, and so we have, from (14),

$$
\int_{C r} \delta\left(B_{r}(y) \cap C\right) d y=\theta_{s} r^{s} \delta(C) \text {. }
$$

Further, for $y \in C^{r}$ we have $B_{r}(y) \subseteq C^{2 r} \subseteq Q$. and hence $B_{r}(y)=B_{r}(y) \cap C^{2 r}$. So we can calculate

$$
\begin{aligned}
\int_{C^{r}} \bar{\delta}\left(B_{r}(y)\right) d y & =\theta_{s} r^{s} \bar{\delta}(C)+\int_{C^{r}} \bar{\delta}\left(B_{r}(y) \cap\left(C^{2 r} \backslash C\right)\right) d y \\
& =\theta_{s} r^{s} \delta(C)+\int_{\left(C^{r} \backslash C\right)} \delta\left(B_{r}(y) \cap\left(C^{2 r} \backslash C\right)\right) d y \\
& \geqq \theta_{s} r^{s} \delta(C)-V\left(C^{r} \backslash C_{r}\right) \theta_{s} r^{s} \\
& =\theta_{s} r^{s}\left\{\bar{\delta}(C)-V\left(C^{r} \backslash C\right)-V\left(C \backslash C_{r}\right)\right\}
\end{aligned}
$$




$$
\geqq \theta_{s} r\left\{\delta(C)-2 r s(1+2 r)^{s}-2 r s\right\}
$$

by Lemmas 1 and 2 . Now, on substituting for $r$, we obtain

$$
\begin{aligned}
\int_{C r} \delta\left(B_{r}(y)\right) d y & \geqq \theta_{s} r^{s}\left\{\delta(C)-\frac{\delta(C)}{4}\left(1+\frac{\delta(C)}{8 s}\right)^{s}\right\} \\
& \geqq \theta_{s} r^{s} \delta(C)\left(1-\frac{1}{4} e^{t}\right) \\
& \geqq \frac{1}{2} \theta_{s} r^{s} \delta(C)=\frac{\theta_{s}}{2(16 s)^{s}}(\delta(C))^{s+1} .
\end{aligned}
$$

So for some $y \in C$,

$$
V\left(C^{r}\right) \bar{\delta}\left(B_{r}(y)\right) \geqq \frac{\theta_{s}}{2(16 s)^{s}}(\bar{\delta}(C))^{s+1} .
$$

Since $V\left(C^{r}\right) \leqq 1$, we therefore have the theorem with $\phi_{1}=\theta_{s} / 2(16)^{s}$.

CASE III: $\delta(C)<0, C^{2 r} \ddagger Q, r=\delta(C) / 16 s$. Now if

then by (15),

$$
\delta\left(C \cap Q_{2 r}\right) \geqq \frac{1}{2} \delta(C) \text {, }
$$

$$
r^{\prime}=\bar{\delta}\left(C \cap Q_{2 r}\right) / 16 s \leqq \delta(C) / 16 s=r,
$$

and so $\left(C \cap Q_{2 r}\right)^{2 r^{\prime}} \subseteq Q$. We could therefore apply case II to $C \cap Q_{2 r}$ instead of $C$, and obtain

$$
|\delta(B)| \geqq \phi_{1}\left|\delta\left(C \cap Q_{2 r}\right)\right|^{s+1} \geqq \phi_{1} 2^{-(s+1)}|\delta(C)|^{s+1} .
$$

Thus the theorem would still be true, but with

$$
\phi_{1}=\theta / 2^{s+2}(16 s)^{s} \text {. }
$$

We can therefore assume that

$$
\delta\left(C \cap\left(Q \backslash Q_{2 r}\right)\right) \geqq \frac{1}{2} \delta(C) .
$$

But now

by Lemma 2 ,

$$
\begin{aligned}
\bar{\delta}\left(Q \backslash Q_{2 r}\right) & =\bar{\delta}\left(C \cap\left(Q \backslash Q_{2 r}\right)\right)+\bar{\delta}\left(\left(Q \backslash Q_{2 r}\right) \backslash C\right) \\
& \geqq \frac{1}{2} \bar{\delta}(C)-V\left(Q \backslash Q_{2 r}\right) \\
& \geqq \frac{1}{2} \bar{\delta}(C)-2 s \cdot 2 r
\end{aligned}
$$

$$
=\bar{\delta}(C) / 4 \text {. }
$$

Hence, as $\delta(A)+\delta(Q \mid A)=0$ for any $A \subseteq Q$, we have

$$
\delta\left(Q_{2 r}\right) \geqq \frac{1}{4} \delta(C) \text {. }
$$


Thus we are reduced to case I, with the convex set $Q_{2 r}$ replacing $C$. So we again obtain the required result, but with constant

$$
\phi_{1}=\frac{\theta_{s}}{2(12 s)^{s}} \cdot \frac{1}{4^{s+1}}=\frac{\theta_{s}}{8(48 s)^{s}} .
$$

This last constant is the smallest obtained in any of the cases, so the theorem is true with $\phi_{1}$ given by (18).

\section{References}

[1] H. G. Eggleston, Convexity, Cambridge Tract no. 47 (C. U. P. 1958).

[2] E. Hlawka, 'Zur Definitionen der Diskrepanz', Acta Arith. 18 (1971), 233-241.

[3] C. J. Smyth, Ph.D. dissertation, University of Cambridge, 1972.

Department of Pure Mathematics and Mathematical Statistics

University of Cambridge

16 Mill Lane

Cambridge CB2 ISB

England 